\title{
Comprehensive Learning Bat Algorithm for Optimal Coordinated Tuning of Power System Stabilizers and Static Var Compensator in power systems
}

\author{
Bousaadia BAADJI ${ }^{\mathrm{a}, *}$; Hamid BENTARZI ${ }^{\mathrm{a}}$; Azzeddine BAKDI ${ }^{\mathrm{b}}$ \\ ${ }^{a}$ Signals and Systems Laboratory, Institute of Electrical and Electronics Engineering, University M'Hamed Bougara \\ of Boumerdes, Avenue of independence, 35000 Boumerdès, Algeria \\ ${ }^{\mathrm{b}}$ Department of Mathematics, University of Oslo, 0851 Oslo, Norway \\ b_baadji@yahoo.com,bentarzi_hamid@yahoo.com*,bkdaznsun@gmail.com
}

\begin{abstract}
This article presents a novel Comprehensive Learning Bat Algorithm (CLBAT) for the optimal coordinated design of Power System Stabilizers (PSSs) and Static Var Compensator (SVC) for damping electromechanical oscillations in multimachine power systems considering wide range of operating conditions. The CLBAT incorporates a new comprehensive learning strategy (CLS) to improve the micro-bats cooperation, location update is also improved to maintain the bats diversity and to prevent premature convergence through a novel adaptive search strategy based on the relative travelled distance. In addition, the proposed elitist learning strategy (ELS) speeds up the convergence during the optimization process and drives the global best solution (gbest) toward promising regions. The superiority of the CLBAT over recent algorithms is first demonstrated via several experiments and comparisons through benchmark functions. The developed algorithm ensures convergence speed, credibility, computational resources, and optimal tuning of PSSs and SVCs of multimachine systems under different operating conditions through eigenanalysis, nonlinear simulation, and performance indices.
\end{abstract}

Keywords - Power System Stabilizer; Static var compensator; Comprehensive learning; Adaptive search strategy; Elitist learning; Optimal control. 


\section{Introduction}

Electromechanical oscillations impose great challenges in modern power systems since they limit the maximum power transfer capability and deteriorate the system stability (Kundur et al. 1994; Klein et al. 1991).PSSs are thus widely employed to damp such electromechanical oscillations and restore the operation stability. However, under some operating conditions, PSSs fail to provide enough damping especially for inter-area oscillation modes. The recent emergence of Flexible AC Transmission Systems (FACTs) provides an alternative solution to improve the system damping (Bian et al. 2016; Shahgholian et al. 2016). Among the various types of FACTs, the Static Var Compensator (SVC) which is one of the most common devices used for this purpose. Although the SVC is basically employed for regulating the bus voltage, several studies demonstrated that it can also boost the system stability (Mondal et al. 2012; Abido et al. 2003).

The simultaneous design of controllers in power systems is based on the optimization of complex non-differentiable problem that generally causes serious challenges for the application of the traditional compensation strategies.

Several analytical approaches, based on traditional control theory, have been used to design robust power system controllers such as Hœo optimization techniques (T.C.Yang 1997), structured singular value (SSV) (Castellanos et al. 2008), and Bilinear matrix inequalities (de Campos et al. 2014). These designs face the common problems of the selection of weighting functions, pole-zero cancellation, and their requirements for higher order controllers which make them less common in applications. Adaptive control techniques were also proposed (Nechadi et al. 2012, Hussein et al. 2010) where the controllers are designed with quickly adjustable parameters according to the changes in the system parameters. However, since power systems are time-varying, the implementation costs of real-time adaptive controllers are high as the on-line parameter identification process is computationally heavy, and particularly if optimal design is considered. Alternatively, Artificial Neural Network (ANN) has been adopted in the design of PSSs (Tofighi et al. 2015, 
Mahabuba et al. 2009). ANN-based controllers can significantly improve the system performance however at the price of their exploding computation capacity with large amounts of training data and long training time. (Khan et al. 2006) employed Fuzzy Logic Control (FLC) for the design of PSSs (Sambariya et al. 2015, Bouchama et al. 2016) to address the inaccuracies and uncertainties in the system model. However, extensive refinement to FLC are required before the application.

Modern studies employ population-based algorithms to overcome these problems and achieve optimal settings for robust performance. Genetic algorithm (Sebaa et al. 2009) was proposed for the optimal tuning of PSSs in multimachine system, Teaching-Learning algorithm with chaotic strategy (Farah et al. 2017) is adopted in the coordinated design of Thyristor-Controlled Series Capacitor (TCSC) and PSSs. The small signal stability of power system is enhanced through the tuning of SVC and TCSC via Particle Swarm Optimization (PSO) algorithm (Mondal et al. 2012). The flower pollination algorithm was employed for robust design of SVC in power system (Abdelaziz et al. 2015). Gravitational Search Algorithm (GSA) was combined with gradient local search method (Peres et al. 2018) and employed for various types of PSSs design. The optimal design of PSSs is proposed via cuckoo search algorithm (Elazim et al. 2016). Bacteria foraging algorithm is adopted for the coordinated tuning of SVC and PSSs (Abd-Elazim et al. 2012). Shuffled frog-leaping algorithm (Darabian et al. 2015) is proposed to improve power system stability through the optimal tuning of SVC. Despite the many research items in the field, an optimal solution to this optimization problem does not exist in a closed form which always allows for future improvements.

Recently, BA algorithm (Yang et al. 2012) is a new meta-heuristic algorithm inspired from the echolocation behaviour of Microbats. The BA employs a varying frequency, with increasing pulse emission rates and decreasing loudness of bats to search and locate the global best solution (gbest). The key advantage of BA is its higher accuracy finding the optimal solution due to the echolocation capacity of its micro-bats which can efficiently find their prey, distinguish it from local candidates, and precisely 
determine its location. However, BA still prone to premature convergence and trapping into local optima, this issue in addition to the unsatisfied balance between exploration and exploitation require further improvements which will be introduced along with novel developments in this article.

Accordingly, variants of the BA have been proposed in attempt to enhance its optimization performance. (Liu et al. 2018)enhanced the BA local search capability based on chaotic initialization of the population, position update via a nonlinear decreasing time factor, and a hybridization with external optimization algorithm. (Meng et al. 2015) proposed the compensation for Doppler Effect in echoes and the foraging habitat of bats by further mimicking the bats behaviour. Four strategies were also proposed in (BahmaniFirouzi et al. 2014) for updating the bat velocities in which an accumulator for each strategy is computed and used to determine the probability of selecting that strategy. (Saad et al. 2018) recently utilized a kriging surrogate model to solve Computationally Expensive Black-Box optimization problems. Despite the several efforts, the possibilities for enhancement are still on, as there is no algorithm that is ultimately perfect as it is indicated by the no free lunch theorem (Ho, Y. C et al. 2002).

The utilization of the information of individuals from the previous iterations for the improvement of metaheuristic algorithms has proved to be an efficient strategy (Wang, et al. 2017). As an example, the modified biography biogeography-based optimization, proposed by (Jalili et al. 2016), adopted a strategy in which elite individuals are transferred from the previous generation to the current one where they were reused in combinations with the new individuals. Bare bones artificial bee Colony (Gao et al. 2015) improves the search ability through gaussian search equation that exploits the information of the best individual to generate a new candidate at the onlooker phase. In this employed bee phase, a parameter adaptation strategy and a fitness-based neighbourhood mechanism exploit the information from the previous search and from the best individuals, respectively. The Comprehensive Learning Particle Swarm Optimizer (CLPSO) (Liang et al. 2006) incorporates a CLS to improve its efficiency via the cooperative learning based 
on the exchange of information from previous iterations between all the particles in the swarm. Motivated by these significant improvements, a novel CLS is developed as an extension to the traditional BA which ignores the use of previous information from other individuals except for the best candidate. A CLBAT algorithm is introduced to improve and control the search memory during the optimization process in which the current location can potentially learn from the previous best locations of all individuals, and not restricted with the global best location as in the original BA. In addition, an ELS is proposed to push gbest out of regions of local optima. Moreover, a new adaptive search strategy is introduced to control the travelled distance of each micro-bat as an indicator of the tendency to either exploit or explore for new solutions which plays a positive role in speeding up the optimization process which is a known limitation of the original BA. The proposed algorithm is first tested against benchmark functions in which it shows superior performance. CLBAT is then employed for the coordinated design of PSSs and SVC controllers for a two-area four-machine (TAFM) system under different operating conditions, the performance of the obtained controllers is evaluated through eigenvalue analysis, nonlinear simulation, and indices where the proposed controllers effectively damp out the electromechanical oscillations and attain robust performance for all the considered conditions.

This article introduces the CLBAT algorithm which is tested against the benchmark test functions then employed for the coordinated design of the PSSs and SVC. The obtained results and relevant comparisons are discussed and the concluding remarks are drawn with proposals for future works.

\section{Problem formulation}

\subsection{Power system modelling}

The power system model is generally described using a set of nonlinear differential equations (Kundur et al. 1994):

$$
\dot{x}=f(x, u)
$$


Where $x$ is the vector of the system states including the generators, loads, and other controllers such as SVCs and PSSs, and $u$ is the vector of the system inputs. A full description of the set of equations governing the system operation are available in the literature (Sauer et al.1998).

For a given operating point, the linearized model is represented in state space approach as:

$\dot{x}=A x+B u$

$A$ is the state matrix that determines the system eigenvalues, and is obtained by $\frac{\delta f}{\delta x}$ at a given operating point, where $B$ is the input matrix that is equal to $\frac{\delta f}{\delta u}$.

The damping ratio $\zeta$ that corresponds to a single eigenvalue $\lambda$ is given by:

$\zeta=\frac{-\operatorname{real}(\lambda)}{\sqrt{\operatorname{real}(\lambda)^{2}+\operatorname{imag}(\lambda)^{2}}}$

This article considers the optimal tuning of PSSs and SVC controllers for such a system to properly allocate the oscillation modes in the complex plane and increase the damping ratios of electromechanical modes.

\subsection{PSS modelling:}

The main function of a PSS is to improve the system stability and mitigate the electromechanical oscillations through modulating the Automatic Voltage Regulator (AVR) output. The conventional PSS (figure 1) is adopted in this work, it is composed of a stabilizer gain $K s$, washout block, and lead-lag compensators. Its input is the rotor speed deviation $(\Delta \omega)$.

The role of $K_{S}$ is to specify the amount of damping injected to mitigate the system oscillations. The washout block is a high pass filter that prevents the DC component of the input signal from affecting the terminal voltage, hence $T w$ is set to $10 \mathrm{~s}$. The two lead-lag blocks characterized by time constants $T_{1}, T_{2}, T_{3}$, and $T_{4}$ (in seconds) are used to compensate for the phase lag between the input and the output of the PSS. 


\subsection{SVC modelling:}

The SVC (figure 2) is a shunt connected static var generator that consists of a fixed capacitor $\boldsymbol{C}$ in parallel with a thyristor-controlled rectifier (TCR). The SVC maintains a fixed bus voltage and boosts the system stability. Its contribution in damping such oscillations is significantly improved by adding an auxiliary controller, usually a lead-lag compensator to the voltage control loop of the SVC.

The block diagram of SVC shown in figure 3 presents the thyristor firing control system characterized by gain $K_{\mathrm{r}}$ and time constant $T_{r}$, and an auxiliary controller characterized by a gain $K_{s v c}$ and two lead-lag blocks. The firing angle of the thyristor adjusts the SVC output which is the equivalent susceptance so as to control the bus voltage.

The state space representation of the SVC controller is therefore given by:

$$
\dot{B}_{s v c}=\frac{1}{T_{r}}\left(-\dot{B}_{s v c}+k_{r}\left(V_{r e f}-V_{t}+V_{s}\right)\right)
$$

\section{Bat Algorithm}

\subsection{Original Bat Algorithm}

BA is a metaheuristic optimization algorithm that has been applied successfully for solving various optimization problems such as power system stabilizers design (Ali et al. 2014), robust design of multiple trailing edge flaps for helicopter vibration reduction (Mallick et al. 2015), the redundancy allocation problem (Talafuse et al. 2016), Maximum Power Point Tracking in photovoltaic systems (Oshaba et al. 2017), and for tuning PI controllers in order to design Load Frequency Controller (LFC) (Abd-Elazim et al. 2016). The echolocation behaviour of its bats gives them the ability not only to localize their prey but also to discriminate it from other objects. Microbats use a type of sonar, called echolocation, to detect prey, they emit a sound pulse with loudness that varies from the loudest when searching for prey to a quieter base when approaching the prey. Most bats use short frequency-modulated signals for echolocation. 
It is formulated by idealizing the echolocation behaviour of bats using the following approximate rules:

(1) All bats use echolocation to sense distance of the prey and obstacles and to discriminate between them.

(2) The bats fly randomly with velocity $v_{i}$ at position $x_{i}$ with a fixed frequency $f_{\min }$, varying wavelength $\lambda$ and loudness $A_{0}$ to search for their prey. They adjust the frequency of the emitted pulses, and the rate of pulse emission $r$ in the range of $[0,1]$, depending on the proximity of their target.

(3) The loudness varies from a large $A_{0}$ to a minimum value $A_{\min }$, while the frequency varies within the range $\left[f_{\min }, f_{\max }\right]$.

\subsubsection{BA Design procedures}

The basic procedure of BA is shown in figure 4, which can be summarized through the following steps: At the initial phase the positions $x_{i}$ and velocities $v_{i}$ of the bats are randomly distributed in the $D$-dimensional search space, and they are updated in each iteration according to the following equations:

$f_{i}=f_{\min }+\left(f_{\max }-f_{\min }\right) \beta$

$v_{i}^{t}=v_{i}^{t-1}+\left(x_{i}^{t}-x_{g}^{t}\right) f_{i}$

$x_{i}^{t}=x_{i}^{t-1}+v_{i}^{t}$

where $\beta \in[0,1]$ is a uniform random vector, $x_{\mathrm{g}}{ }^{\mathrm{t}}$ is the current gbest, while $f_{\max }$ and $f_{\min }$ are respectively the values of the maximum and minimum frequencies.

For each bat, its pulse rate is less than a uniform random number within $[0,1]$. The local search using random walk is performed to generate a new solution around the current gbest.

$x_{\text {new }}=x_{g}^{t}+\varepsilon A^{t}$ 
Where $\varepsilon \in[-1,1]$ represents a uniform random number, and $A^{t}$ stands for the mean of the loudness of all bats at iteration $t$. As the number of iterations increases, the bats get closer to their target, and the loudness is decreased while the pulse rate emission is increased. Hence the update for those parameters is given as:

$A_{k}^{t+1}=\alpha A_{k}^{t}$

$r_{k}^{t+1}=r_{k}^{o}\left(1-e^{-\gamma t}\right)$

Where $\alpha$ is a constant selected such that $0<\alpha<1$, and $\gamma$ is a positive constant.

\subsection{Comprehensive learning Bat Algorithm}

\subsubsection{Comprehensive learning strategy:}

The original BA relies only on its gbest to update the bats velocities at each iteration, and eventually all bats are attracted to the region of gbest that is expected to be the global optimum. However, in case gbest is stuck in local optimum region, the bats get trapped in that local optima. To overcome this deficiency, the CLBAT employs an information sharing strategy known as CLS (Liang et al. 2006) in which the information stored about the bats previous best locations serve to improve the performance by increasing the diversity through the adopted velocity updating strategy:

$$
v_{d i}^{t}=w \cdot v_{d i}^{t-1}+r_{d i} \cdot d \cdot\left(x_{d i}^{t}-b x_{d i}^{t}\right) f_{d i}
$$

Where $v_{d i}^{t}$ is the velocity of the $d_{t h}$ dimension of the $i_{t h}$ bat at iteration $t$, similarly $x_{d i}^{t}$ and $b x_{d i}^{t}$ are the current location and the best location of the $d^{\text {th }}$ dimension of the $i^{\text {th }}$ bat at iteration $t$, and $f_{d i}$ is the frequency of that dimension.

$r_{d i}$ is a random number uniformly distributed between 1 and 0 , and $\boldsymbol{d}$ is an inertia weight.

$W$ is the inertia weight which is decreased linearly from $W_{\max }$ to $W_{\min }$ :

$$
w=\left(w_{\max }-w_{\min }\right) \cdot\left(1-\frac{i t e r}{i t e r_{\max }}\right)+w_{\min }
$$


$W$ is used to balance between global and local search. It is started with large inertia weight to rapidly direct the algorithm toward global search, and decreases as the number of iterations increase to encourage exploitation during the convergence toward the global solution.

To illustrate the advantage of the CLBAT, consider the problem of minimizing a function $f(X)$ with dimension $D$, where $X=\left(x_{1}, x_{2}, \ldots ., x_{D}\right)$, and $X_{G}=\left(x_{G 1}, x_{G 2}, \ldots, x_{G D}\right)$ denote a solution in the search space and the global optimum, respectively. An arbitrary solution $X_{c}$ with poor fitness $f\left(X_{c}\right)$ could discover the $i^{\text {th }}$ dimension's solution such that $x_{C i}=x_{G i}$. In order to keep and transfer this beneficial information, the CLBAT enables the sharing of information among bats through the CLS that is based on two features. (i) Only the sharing of previous best information is allowed. (ii) A learning probability $P_{c i}$ controls the update of velocity at a dimension $d$ using the dimension of a randomly selected best location $b x_{T d}$ in the case where $P_{c i}$ is less than a randomly generated number, otherwise it is updated using its own best location $b x_{i d}$. This velocity update strategy is demonstrated in Figure 5.

The learning probability is crucial in the determination of CLBAT performance, and it is given as:

$P_{c}=\left(P_{\max }-P_{\min }\right) \cdot\left(1-\frac{i t e r}{i t e r_{\max }}\right)^{2}+P_{\min }$

The nonlinear $P c$ is more adequate for proper balance between the exploration and the exploitation states of the CLBAT. At the early stage, relatively high values of $P c$ encourage the micro-bats to learn from their own previous information through the use of their best locations because the CLBAT is in the exploration phase (High $w$ ) since the sharable information is very limited at this stage. In the final stage, however, the relatively low $P c$ encourages information sharing in which a bat will more probably learn from other bats best locations because the CLBAT is in the convergence state where more useful information is discovered, especially those about the global optimum at some dimensions. Therefore, the controlled information sharing among the bats allows useful propagation within the population. 


\subsubsection{New search Method}

The conventional search strategy of the BA is locally limited around gbest based on the mean of the loudness. Due to this high dependence however, the loudness control of the search radius is not sufficient to speed up the convergence rate of the algorithm. Although the distribution of the bats new locations varies dynamically during the optimization process, the new locations are often far from the old locations at the exploration state, and very close at the final stage, due to the adopted position update strategy in the CLBAT. Hence the difference between the bat new and old location has the same behaviour as the loudness as it is large at the beginning and slowly decreases during the optimization process. The new search method is suitable to adaptively refine gbest for exploration and convergence states, the new search protocol is defined as:

$N x_{i}^{t+1}=x_{g}^{t}+A_{i} \cdot \operatorname{rand} 1 .\left(N x_{i}^{t}-x_{i}^{t}\right)$

where $N x_{i}^{t}$ represents the bat's new location, $x_{g}^{t}$ is the current gbest, and rand 1 is a random number drawn from a standard normal distribution.

The new search strategy adaptively controls the search around gbest depending on the last travelled distance from the bat's old location to its new location. Since $N x_{i}^{t}$ is far from $x_{i}^{t}$ in the exploration phase, the search around gbest is performed with large travelled steps and more potential location may be discovered. In contrast, the distance between $N x_{i}^{t}$ and $x_{i}^{t}$ is minimal at the final stage and the search only refines gbest.

\subsubsection{Modified Elitist learning strategy:}

A modified ELS (figure 6), is adopted as a jumping out mechanism that updates gbest at randomly selected dimensions to move it to better regions and help escape local optima. One dimension of a gbest solution is selected randomly to undergo modifications in order to preserve the main structure of gbest, since many dimensions of gbest contains the information about the global optimum. The modified ELS is given as:

$$
N x_{g}^{d}=x_{g}^{d}+\operatorname{rand} 2 .\left(x_{\text {max }}^{d}-x_{\text {min }}^{d}\right)
$$


where rand 2 is a random number from a uniform distribution within [-1, 1].

It is worth noting that in the ELS proposed (Zhan et al. 2009), $N x_{g}^{d}$ replaces gbest if its fitness is better otherwise it replaces the worst fitness particle $X_{w}$. Notice that this strategy may slow down the convergence if the fitness of $N x_{g}$ is worse than that of $X_{w}$, for that reason $N x_{g}$ is kept in the modified ELS only if its fitness is better than the fitness of gbest or $X_{w}$.

\section{Test functions}

The performance of the developed CLBAT algorithm is first tested on a set of benchmark functions with various properties in order to assess its ability in exploring the region of search and jumping over regions of a local optimum. Table 1 lists the details of each test function with its corresponding name, optimization function, range of its search space, and the maximum tolerance value to accept an optimization solution.

The resulting performance of the CLBAT is also compared with Artificial Bee Colony (ABC) algorithm (Karaboga et al. 2007), original BA Algorithm (Yang et al. 2012), and Biogeography-Based Optimization (BBO) (Simon, 2008). For a robust and fair comparison of results, the algorithms are tested for 30 independent runs for a sufficient maximum number of fitness evaluations set to 120.000 and with a population size of 40 . The mean and standard deviation of the proposed algorithms are presented in table 2. CLBAT outperforms other algorithms across all the test functions since it reaches accurately the global optimum of functions $\left(f_{1}-f_{5}\right)$ for all the runs with zero mean and standard deviation results. For $\left(f_{6^{-}} f_{11}\right)$ the accuracy is very high and much better compared to the other algorithms as the mean is very close to the global $f_{\min }$. Specifically, the results demonstrate that the CLBAT is able to jump out local solutions through the embedded CLS, modified ELS, and adaptive search strategies.

Table 1. The test numerical functions.

\begin{tabular}{|c|c|c|c|c|}
\hline Name & Formula & Range & Global $f_{\text {min }}$ & Accept \\
\hline Sphere & $f_{1}(x)=\sum_{i=1}^{D} x_{i}^{2}$ & {$[-100,100]$} & 0 & $1 e^{-2}$ \\
\hline Zakharov & $f_{2}(x)=\sum_{i=1}^{D} x_{i}^{2}+\left(\sum_{i=1}^{D} 0.5 x_{i}\right)^{2}+\left(\sum_{i=1}^{D} 0.5 x_{i}\right)$ & {$[-10,10]$} & 0 & $1 e^{-2}$ \\
\hline
\end{tabular}




\begin{tabular}{|c|c|c|c|c|}
\hline Sum square & $f_{3}(x)=\sum_{i=1}^{D}\left(i x_{i}\right)^{2}$ & {$[-100,100]$} & 0 & $1 e^{-5}$ \\
\hline Rastrigin & $\left.f_{4}(x)=\sum_{i=1}^{D}\left(x_{i}^{2}-10 \cos \left(2 \pi x_{i}\right)\right)+10\right)$ & {$[-5.12,5.12]$} & 0 & $1 e^{-}-5$ \\
\hline $\begin{array}{l}\text { Non-continuous } \\
\text { Rastrigin }\end{array}$ & $\begin{array}{l}\left.f_{5}(x)=\sum_{i=1}^{D}\left(y_{i}^{2}-10 \cos \left(2 \pi y_{i}\right)\right)+10\right) \\
y_{i}=\left\{\begin{array}{l}x_{i} \quad\left|x_{i}\right|<0.5 \\
\operatorname{round}\left(2 x_{i}\right) / 2 \quad\left|x_{i}<0.5\right| \geq 0.5\end{array}\right.\end{array}$ & {$[-5.12,5.12]$} & 0 & $1 e-5$ \\
\hline Rosenbrock & $f_{6}(x)=\sum_{i=1}^{D-1}\left(100\left(x_{i}^{2}-x_{i+1}\right)^{2}+\left(x_{i}^{2}-1\right)^{2}\right)$ & {$[-2.048,2.048]$} & 0 & 5 \\
\hline Schwefel 2.21 & $f_{7}(x)=\max \left\{\left|x_{i}\right|, 1 \leq i \leq D\right\}$ & {$[-100,100]$} & 0 & $1 e^{-2}$ \\
\hline Schwefel 2.22 & $f_{8}(x)=\sum_{i=1}^{D}\left|x_{i}\right|+\prod_{i=1}^{D}\left|x_{i}\right|$ & {$[-10,10]$} & 0 & $1 e^{-2}$ \\
\hline Levy & $\begin{array}{l}\begin{array}{l}f_{9}(x)=\sin ^{2}\left(\pi y_{i}\right)+\sum_{i=1}^{D-1}\left(y_{i}-1\right)^{2}\left(1+10 \sin ^{2}\left(\pi y_{i}+1\right)\right) \\
\quad+\left(y_{D}-1\right)^{2}\left(1+\sin ^{2}\left(2 \pi y_{D}\right)\right)\end{array} \\
y_{i}=1+\frac{1}{4}\left(x_{i}-1\right)\end{array}$ & {$[-10,10]$} & 0 & $1 \mathrm{e}-5$ \\
\hline Penalized 1 & $\begin{aligned} f_{10}(x)= & \frac{\pi}{D}\left[10 \sin ^{2}\left(\pi y_{1}\right)+\sum_{i=1}^{D-1}\left(y_{i}-1\right)^{2}\left(1+10 \sin \left(\pi y_{i+1}\right)\right)+\left(y_{D}-1\right)^{2}\right. \\
& \left.+\sum_{i=1}^{D} u\left(x_{i}, 10,100,4\right)\right] \\
y_{i}=1+ & \frac{1}{4}\left(x_{i}+1\right) ; u_{x_{i, a}, k, m}= \begin{cases}k\left(x_{i}-a\right)^{m} & x_{i}>a \\
0 & -a \leq x_{i} \leq a \\
k\left(-x_{i}-a\right)^{m} & x_{i}<-a\end{cases} \end{aligned}$ & {$[-50,50]$} & 0 & $1 e-5$ \\
\hline Penalized 2 & $\begin{aligned} f_{11}(x)= & \frac{\pi}{D}\left[10 \sin ^{2}\left(3 \pi x_{1}\right)+\sum_{i=1}^{D-1}\left(x_{i}-1\right)^{2}\left(1+10 \sin \left(3 \pi x_{i+1}\right)\right)+\left(x_{D}-1\right)^{2}\right. \\
& \left.+\sum_{i=1}^{D} u\left(x_{i}, 5,100,4\right)\right]\end{aligned}$ & {$[-50,50]$} & 0 & $1 e-5$ \\
\hline
\end{tabular}

Table 2. The results of CLBAT compared to the state-of-the art algorithms.

\begin{tabular}{|c|c|c|c|c|c|}
\hline Name & & CLBAT & $\mathrm{ABC}$ & BA & BBO \\
\hline \multirow{2}{*}{ Sphere } & Mean & $00 \mathrm{e} 00$ & $4.6103 \mathrm{e}-16$ & $1.7617 \mathrm{e}-06$ & $4.5731 \mathrm{e}-09$ \\
\hline & StdDev & 00 & $4.9036 \mathrm{e}-17$ & $4.6416 \mathrm{e}-07$ & $5.2354 \mathrm{e}-09$ \\
\hline \multirow[t]{2}{*}{ Zakharov } & Mean & $00 \mathrm{e} 00$ & $5.0351 \mathrm{e}-16$ & $6.3080 \mathrm{e}-05$ & $1.2455 \mathrm{e}-09$ \\
\hline & StdDev & $00 \mathrm{e} 00$ & $7.1333 \mathrm{e}-17$ & $1.6868 \mathrm{e}-05$ & $4.8086 \mathrm{e}-09$ \\
\hline \multirow{2}{*}{ Sum square } & Mean & 00e00 & $5.1222 \mathrm{e}-16$ & $5.2112 \mathrm{e}-05$ & $1.3093 \mathrm{e}-07$ \\
\hline & StdDev & 00e00 & $4.2745 \mathrm{e}-17$ & $1.3938 \mathrm{e}-05$ & $8.6187 \mathrm{e}-08$ \\
\hline \multirow[t]{2}{*}{ Rastrigin } & Mean & 00e-00 & $4.1685 e-14$ & $1.4213 \mathrm{e} 02$ & $2.0187 \mathrm{e}-06$ \\
\hline & StdDev & 00e-00 & $2.5567 \mathrm{e}-14$ & $3.2932 \mathrm{e} 01$ & $2.3970 \mathrm{e}-06$ \\
\hline \multirow{2}{*}{$\begin{array}{l}\text { Non-continuous } \\
\text { Rastrigin }\end{array}$} & Mean & $00 \mathrm{e} 00$ & $00 \mathrm{e} 00$ & $1.7249 \mathrm{e} 02$ & $4.2680 \mathrm{e} 00$ \\
\hline & StdDev & $00 \mathrm{e} 00$ & $00 \mathrm{e} 00$ & $4.9104 \mathrm{e} 01$ & $1.3620 \mathrm{e} 00$ \\
\hline \multirow[t]{2}{*}{ Rosenbrock } & Mean & $2.658 \mathrm{e}-01$ & $3.6731 \mathrm{e} 00$ & $8.394 \mathrm{e}-01$ & $2.1166 \mathrm{e} 01$ \\
\hline & StdDev & $1.0114 \mathrm{e} 00$ & $2.5191 \mathrm{e} 00$ & $1.6207 \mathrm{e} 00$ & $4.6231 \mathrm{e} 00$ \\
\hline \multirow{2}{*}{ Schwefel 2.22} & Mean & $1.4767 \mathrm{e}-46$ & $1.2455 \mathrm{e}-15$ & $2.672 \mathrm{e}-01$ & $3.9218 \mathrm{e} 03$ \\
\hline & StdDev & $6.1958 \mathrm{e}-46$ & $9.7600 \mathrm{e}-17$ & $7.690 \mathrm{e}-01$ & $5.0525 \mathrm{e} 02$ \\
\hline \multirow{2}{*}{ Schwefel 2.21} & Mean & $8.5432 \mathrm{e}-115$ & $2.4731 \mathrm{e} 01$ & $2.1494 \mathrm{e} 01$ & $1.26 \mathrm{e}-02$ \\
\hline & StdDev & $4.1846 \mathrm{e}-114$ & $3.2802 \mathrm{e} 00$ & $7.1633 \mathrm{e} 00$ & $2.80 \mathrm{e}-03$ \\
\hline \multirow{2}{*}{ Levy } & Mean & $1.4998 \mathrm{e}-32$ & $4.4509 \mathrm{e}-16$ & $3.4239 \mathrm{e} 01$ & $1.6999 \mathrm{e} 00$ \\
\hline & StdDev & $1.1135 e-47$ & $6.9267 e-17$ & $1.1179 \mathrm{e} 01$ & $1.4384 \mathrm{e} 00$ \\
\hline \multirow{2}{*}{ Penalized 1} & Mean & $1.5705 \mathrm{e}-32$ & $4.5600 \mathrm{e}-16$ & $1.07599 \mathrm{e} 01$ & $1.8114 \mathrm{e}-11$ \\
\hline & StdDev & $5.5674 \mathrm{e}-48$ & $7.5544 \mathrm{e}-17$ & $1.08950 \mathrm{e} 01$ & $4.7946 \mathrm{e}-11$ \\
\hline
\end{tabular}




\section{Controllers Design using CLBAT algorithm:}

The modes of oscillations in a linear system are related to its eigenvalues. To improve the power system stability, a multi-objective function is employed to relocate all the eigenvalues within the D-contour (AbdelMagid et al. 2003). The latter is characterized by $\delta_{i j} \leq \delta_{o}$, and $\zeta_{i j} \geq \zeta_{o}$, as shown in Figure 7. The values of $\delta_{O}, \zeta_{O}$ , and the weighting factor $q$ are empirically set to $-2.0,0.25$, and 10 respectively based on the system under study to ensure sufficient damping to the electromechanical oscillations. The PSSs and SVC parameters are simultaneously tuned using CLBAT to shift all modes within the D-contour over a given range of operating conditions to guarantee a well damped response over that specified range.

The design problem is formulated based on the aforementioned criterion as to minimize the multi-objective function $\boldsymbol{J}$ :

$J=\sum_{j=1}^{n p} \sum_{\delta_{i j}<\delta_{o}}\left(\delta_{o}-\delta_{i j}\right)^{2}+q \sum_{j=1}^{n p} \sum_{\zeta_{i j} \leq \zeta_{o}}\left(\zeta_{o}-\zeta_{i j}\right)^{2}$

Subjected to the following constraints:

$$
\begin{aligned}
& 0.01 \leq K_{s i} \leq 100 \\
& 0.01 \leq T_{1 i} \leq 2 \\
& 0.01 \leq T_{2 i} \leq 2 \\
& 0.01 \leq T_{3 i} \leq 2 \\
& 0.01 \leq T_{4 i} \leq 2
\end{aligned}
$$

Where $i$ denotes the number of controllers which is five in this study, $\boldsymbol{n p}$ is the number of operating conditions considered in the design process, and $\sigma_{i j}$ and $\zeta_{i j}$ are respectively the real part and the damping ratio of the $i^{\text {th }}$ eigenvalue of the $j^{\text {th }}$ operating point. 


\section{Results and simulation}

The TAFM system as shown in figure 8, is a well-known benchmark for power system controllers design, test, and comparisons of the damping efficiency. The system is composed of two areas linked through a weak transmission line that allows a 400 MW active power to flow from area 1 to area 2 . The data for the system is available in (Kundur et al. 1994).

In controllers design, four operating conditions (Table 3) are considered (Eslami et al. 2012) to achieve a robust performance during frequent disturbances that occur during the system operation.

Table 3. Operating conditions.

\begin{tabular}{|l|l|}
\hline Operating condition & Description \\
\hline Case 1 & Base case (All lines in service) \\
\hline Case 2 & Single line between 7 and 8 out of service \\
\hline Case 3 & Single line between 8 and 9 out of service \\
\hline Case4 & Single line between 7 and 9 out of service \\
\hline
\end{tabular}

\subsection{Optimal SVC location selection:}

The optimal location of the SVC is selected using the effect of line outage on the system voltages (AbdElazim et al. 2012). As indicated in Table 4, the voltage at Bus 8 is largely affected by the line outages, especially in case 4 where the voltage at bus drops significantly from 0.9647 pu to $0.787828 \mathrm{pu}$. Hence, bus 8 is the suitable location for installing the SVC, this accords with the results reported in (Martins et al. 1989). The line current between buses 9 and 10 is selected as input for SVC since it has high observability to the inter-area mode (Kundur et al. 1994).

Table 4. Effect of line outage on load bus voltages.

\begin{tabular}{|l|l|l|l|l|}
\hline Cases & Base case & Outage of line 7-8 & Outage of line 8-9 & Outage of line 7-9 \\
\hline Bus 5 & 1.0079 & 1.0036 & 1.004 & 0.99702 \\
\hline Bus6 & 0.98156 & 0.971 & 0.97199 & 0.95499 \\
\hline Bus 7 & 0.9672 & 0.94821 & 0.95 & 0.91952 \\
\hline Bus 8 & 0.9647 & $\mathbf{0 . 9 2 4 2}$ & $\mathbf{0 . 9 0 9 2 5}$ & $\mathbf{0 . 7 8 7 2 8}$ \\
\hline Bus 9 & 1.0044 & 0.99217 & 0.9905 & 0.96632 \\
\hline Bus 10 & 1.0086 & 1.0017 & 1.0008 & 0.98727 \\
\hline Bus 11 & 1.0228 & 1.0201 & 1.0197 & 1.0143 \\
\hline
\end{tabular}




\subsection{PSS and SVC tuning using CLBAT algorithm:}

The CLBAT algorithm is applied to simultaneously tune the 25 parameters of the four PSSs installed in the generators and the SVC. All operating conditions are considered in the design stage to guarantee an optimal damping during all possible conditions and ensure the robustness of the controlled system during variations. The obtained parameters of the controllers are listed in table 5. Besides CLBAT superior accuracy and robustness, CLBAT controllers gains are lower than those of BA, which is another practical advantage in the realization of the controllers.

Table 5. Parameters of the proposed controllers for CLBAT and BA algorithms.

\begin{tabular}{|l|l|l|l|l|l|l|l|l|l|l|}
\hline & \multicolumn{9}{|l}{ CLBAT } & \multicolumn{1}{l|}{ BA } \\
\hline & Ks & $\mathrm{T}_{1}$ & $\mathrm{~T}_{2}$ & $\mathrm{~T}_{3}$ & $\mathrm{~T}_{4}$ & $\mathrm{Ks}$ & $\mathrm{T}_{1}$ & $\mathrm{~T}_{2}$ & $\mathrm{~T}_{3}$ & $\mathrm{~T}_{4}$ \\
\hline SVC & 1.0025 & 0.0122 & 0.0227 & 0.0122 & 1.4295 & 2.1800 & 0.0206 & 1.9122 & 0.0275 & 0.9218 \\
\hline G1 & 25.3641 & 1.4867 & 1.9919 & 1.9895 & 0.0113 & 96.2402 & 0.5174 & 1.6257 & 1.1438 & 0.9844 \\
\hline G2 & 7.8495 & 1.9972 & 1.8867 & 1.9947 & 0.0211 & 34.6806 & 0.6349 & 1.1051 & 1.7734 & 0.0206 \\
\hline G3 & 11.0329 & 0.0693 & 0.0328 & 1.9988 & 0.0456 & 98.6927 & 0.4037 & 0.1951 & 1.9751 & 1.5256 \\
\hline G4 & 20.0696 & 1.9911 & 1.9914 & 1.9972 & 0.0125 & 40.7534 & 0.9672 & 0.7700 & 1.2238 & 0.0206 \\
\hline
\end{tabular}

\subsubsection{Optimization performance statistics}

To explore the reliability of each algorithm to minimize the given objective function, the experiment was repeated in 10 runs. The best, worst, and average costs at each iteration based on 10 runs are indicated in figure 9. The statistics in this section are obtained based on an ordinary computer of 4 CPUs of 3.2 GHZ. Optimal design of the controllers' parameters requires the observation of the system behaviour and it is done in an offline stage; hence the optimality of the solution is more important compared to the optimization time. The running time for one iteration of the CLBAT algorithm is $39.0262 \mathrm{~s}$ versus $19.7331 \mathrm{~s}$ for the BA. However, it is the call of the Power system analysis toolbox (PSAT) that is computationally heavy, and especially for the CLBAT algorithm (39.0248 s) compared to BA (19.7325 s) due to the introduced improvements. This time is mainly due to the communication and parameter settings for the PSAT as well as the load flow and eigenanalysis operations. Notice that the PSAT is called according to the population for 
BA, and twice that number for CLBAT. The real average computation time of the optimization process per iteration is really negligible compared to the PSAT computations, this is $1.4 \mathrm{~ms}$ for CLBAT and $0.6 \mathrm{~ms}$ for BA. However, the CLBAT algorithm successfully reaches the desired settings for the controllers with optimum required solution while the best fitness value of the BA is 1.7917. CLBAT is further more reliable in solving the optimization problem, all the 10 runs successfully converge to solution as depicted.

\subsection{Eigen Analysis:}

The eigenvalues of the system without the controllers are listed in table 6 for all the operating conditions. It can be clearly seen that the highlighted inter-area mode is unstable since it has negative damping ratio for all cases. Moreover, the two local modes are stable but close to the margin of the s-plane with insufficient damping ratios around $12 \%$ and $8 \%$ for all cases. The proposed controllers greatly improve the damping as indicated by the resulting eigenvalues as shown in table 7. Moreover, the CLBAT-tuned controllers (CLBAT-C) outperform the original BA-tuned controllers (BA-C) for all cases and for all electromechanical modes. Particularly, the inter-area mode is successfully stabilized with an acceptable damping ratio greater than $58 \%$ for all cases. The damping of the local modes is further increased by CLBAT-C above $50 \%$ for all cases compared to just below $32 \%$ achieved by the BA-C. Moreover, the damping of the inter-area mode with CLBAT-C is better than the state-of-the-art results in (Eslami et al. 2012) for the same system and operating conditions.

Table 6. The open loop system eigenvalues, damping ratios, and frequencies.

\begin{tabular}{|l|l|l|l|}
\hline & Eigenvalues & Damping ratio\% & Frequency $(\mathrm{Hz})$ \\
\hline & $\mathbf{0 . 0 0 8 5} \pm \mathbf{j} \mathbf{3 . 2 8 5 8}$ & $\mathbf{- 0 . 2 5 7 0}$ & $\mathbf{0 . 5 2 3 0}$ \\
Case 1 & $-0.8451 \pm \mathbf{j} 6.5551$ & 12.7870 & 1.0430 \\
& $-0.5353 \pm \mathbf{j} 6.6360$ & 8.0400 & 1.0560 \\
\hline & $\mathbf{0 . 1 8 8 3} \pm \mathbf{j} \mathbf{2 . 4 6 1 9}$ & $\mathbf{- 7 . 6 2 8 0}$ & $\mathbf{0 . 3 9 2 0}$ \\
Case 2 & $-0.8531 \pm \mathbf{j} 6.5426$ & 12.9300 & 1.0410 \\
& $-0.5394 \pm \mathbf{j} 6.5892$ & 8.1590 & 1.0490 \\
\hline & $\mathbf{0 . 1 9 1 5} \pm \mathbf{j} \mathbf{2 . 4 6 0 1}$ & $\mathbf{- 7 . 7 6 1 0}$ & $\mathbf{0 . 3 9 2 0}$ \\
Case 3 & $-0.8541 \pm \mathbf{j} 6.5399$ & 12.9500 & 1.0410 \\
& $-0.5385 \pm \mathbf{j} 6.5917$ & 8.1430 & 1.0490 \\
\hline Case 4 & $\mathbf{0 . 3 9 7 4} \pm \mathbf{j} \mathbf{1 . 3 4 3 5}-$ & $\mathbf{- 2 8 . 3 6 5 0}$ & $\mathbf{0 . 2 1 4 0}$ \\
\hline
\end{tabular}




\begin{tabular}{|l|l|l|l|}
\hline & $0.8455 \pm \mathbf{j} 6.5270-$ & 12.8470 & 1.0390 \\
& $0.5434 \pm \mathbf{j} 6.5383$ & 8.2820 & 1.0410 \\
\hline
\end{tabular}

Table 7. The Eigenvalues, damping ratios, and frequencies of the system with PSSs and SVC tuned by CLBAT and BA.

\begin{tabular}{|l|l|l|l|l|l|l|}
\hline & \multicolumn{3}{|c|}{ CLBAT } & \multicolumn{3}{c|}{ BA } \\
\cline { 2 - 7 } & Eigenvalues & Damping ratio\% & Frequency $(\mathrm{Hz})$ & Eigenvalues & Damping ratio\% & Frequency $(\mathrm{Hz})$ \\
\hline \multirow{3}{*}{ Case1 } & $\mathbf{- 3 . 2 5 0 1} \pm \mathbf{j} \mathbf{4 . 0 1 5 2}$ & $\mathbf{6 2 . 9 1 6 0}$ & $\mathbf{0 . 6 3 9 0}$ & $\mathbf{- 2 . 0 7 2 8} \pm \mathbf{j} \mathbf{2 . 9 0 6 6}$ & $\mathbf{5 8 . 0 6 2 0}$ & $\mathbf{0 . 4 6 3 0}$ \\
& $-7.2855 \pm \mathbf{j} 9.3981$ & 61.2680 & 1.4960 & $-2.0197 \pm \mathbf{j} 9.9362$ & 19.9190 & 1.5810 \\
& $-6.5611 \pm \mathbf{j} 9.9040$ & 55.2270 & 1.5760 & $-3.8776 \pm \mathbf{j} 11.5054$ & 31.9370 & 1.8310 \\
\hline \multirow{3}{*}{ Case2 } & $\mathbf{- 3 . 7 4 2 1} \pm \mathbf{j} \mathbf{2 . 9 4 9 0}$ & $\mathbf{7 8 . 5 4 3 0}$ & $\mathbf{0 . 4 6 9 0}$ & $\mathbf{- 2 . 0 0 7 9} \pm \mathbf{j} \mathbf{3 . 1 6 6 7}$ & $\mathbf{5 3 . 5 5 0 0}$ & $\mathbf{0 . 5 0 4 0}$ \\
& $-7.7259 \pm \mathbf{j} 9.3368$ & 63.7510 & 1.4860 & $-2.7296 \pm \mathbf{j} 10.0837$ & 26.1290 & 1.6050 \\
& $-6.3177 \pm \mathbf{j} 9.6433$ & 54.8010 & 1.5350 & $-3.2763 \pm \mathbf{j} 10.8879$ & 28.8150 & 1.7330 \\
\hline \multirow{3}{*}{ Case3 } & $\mathbf{- 2 . 3 8 4 9} \pm \mathbf{j} \mathbf{3 . 3 0 7 7}$ & $\mathbf{5 8 . 4 8 4 0}$ & $\mathbf{0 . 5 2 6 0}$ & $\mathbf{- 1 . 4 5 5 4} \pm \mathbf{j} \mathbf{2 . 7 2 3 7}$ & $\mathbf{4 7 . 1 2 7 0}$ & $\mathbf{0 . 4 3 3 0}$ \\
& $-6.8595 \pm \mathbf{j} 9.2610$ & 59.5200 & 1.4740 & $-2.4959 \pm \mathbf{j} 9.4909$ & 25.4330 & 1.5110 \\
& $-6.3334 \pm \mathbf{j} 10.098$ & 53.1320 & 1.6070 & $-3.6723 \pm \mathbf{j} 11.2435$ & 31.0470 & 1.7890 \\
\hline \multirow{3}{*}{ Case4 } & $\mathbf{- 2 . 5 7 7 5} \pm \mathbf{j} \mathbf{2 . 7 2 2 8}$ & $\mathbf{6 8 . 7 4 5 0}$ & $\mathbf{0 . 4 3 3 0}$ & $\mathbf{- 0 . 8 5 8 6} \pm \mathbf{j} \mathbf{3 . 7 0 6 1}$ & $\mathbf{2 2 . 5 7 0 0}$ & $\mathbf{0 . 5 9 0 0}$ \\
& $-6.0772 \pm \mathbf{j} 9.4128$ & 54.2410 & 1.4980 & $-1.5742 \pm \mathbf{j} 7.8216$ & 19.7310 & 1.2450 \\
& $-7.4849 \pm \mathbf{j} 9.5150$ & 61.8270 & 1.5140 & $-2.5815 \pm \mathbf{j} 11.1819$ & 22.4940 & 1.7800 \\
\hline
\end{tabular}

\subsection{Nonlinear time-domain simulation:}

Nonlinear simulation is performed on the nonlinear system to evaluate the performance of the proposed controllers considering a small disturbance of a step increase of $0.1 \mathrm{pu}$ in the reference voltage (Wang et al. 2018) of generator 1 at $2 \mathrm{~s}$ with duration of $100 \mathrm{~ms}$ for all the four operating cases, in addition to 3-cycle three-phase fault applied at bus 9 for all cases that is considered as a large disturbance.

Figure 10 illustrates that the CLBAT-Cs outperform the BA-Cs in terms of the oscillations overshoot and the settling times for all the operating cases under the considered small disturbance.

Figure 11 depicts the response of $\omega_{13}$ during 3-cycle three-phase faults at bus 9 for all cases. While both BA$\mathrm{C}$ and CLBAT-C maintain the system stability and restore the destined operation, the CLBAT-Cs improve the damping compared to the BA-Cs. Superior damping results are achieved in terms of the smallest oscillation amplitude, rapid damping, and short settling time. 
Moreover these results are consistent with the eigenvalue analysis of the system, and further demonstrate the capability of CLBAT-Cs to provide high damping to electromechanical oscillations.

To further validate the performance of the proposed controllers, a different operating condition is considered that corresponds to light loading condition in which the transferred power from area 1 to area 2 is decreased to $3.5086 \mathrm{pu}$, then a 3 - cycle three phase fault is applied on bus 9 at $t=1 \mathrm{~s}$.

Figure 12 confirms the superiority of the CLBAT-Cs since the settling time and maximum overshoot are smaller than those of the system response with the BA-Cs.

\subsection{Performance indices}

The robustness of the CLBAT-Cs is measured based on the Integral of the Time-Weighted Absolute Error (ITAE), and the integral Square Error (ISE):

$$
\begin{aligned}
& I T A E=\int_{t=0}^{T s i m} t\left|\Delta \omega_{13}(t)\right| d t \\
& I S E=\int_{t=0}^{T_{\text {sim }}}\left|\Delta \omega_{13}(t)\right|^{2} d t
\end{aligned}
$$

Where, Tsim is the simulation time of the system, set as $10 \mathrm{~s}$. The smaller the values of these indices are, the better the system response is.

Table 8. Performance indices for 3-cycle three phase fault for CLBAT-Cs and BA-Cs.

\begin{tabular}{|l|l|l|l|l|}
\hline \multirow{2}{*}{ case } & \multicolumn{2}{|l|}{ ISE } & \multicolumn{2}{l|}{ ITAE } \\
\cline { 2 - 5 } & CLBAT-Cs & BA-Cs & CLBAT-Cs & BA-Cs \\
\hline Case 1 & $2.4334 \mathrm{e}-07$ & $3.8104 \mathrm{e}-07$ & 0.0014 & 0.0027 \\
\hline Case 2 & $4.4770 \mathrm{e}-07$ & $4.5871 \mathrm{e}-07$ & 0.0021 & 0.0027 \\
\hline Case 3 & $2.8188 \mathrm{e}-07$ & $5.1877 \mathrm{e}-07$ & 0.0013 & 0.0036 \\
\hline Case 4 & $1.4475 \mathrm{e}-06$ & $3.1808 \mathrm{e}-06$ & 0.0058 & 0.0077 \\
\hline
\end{tabular}


The values of the two integral performance criteria in Table 8 demonstrate the superiority of the developed CLBAT-optimized designs of the controllers over their BA-based counterparts in maintaining an optimal response of the power system during the different cases.

\section{Conclusion}

In this article, the coordinated tuning of PSSs and SVC over a wide range of operating conditions for a TAFM system using a new developed CLBAT algorithm is proposed. The significant contributions of the CLBAT algorithm include a new CLS, a modified ELS, and the utilization of adaptive search mechanism. This greatly enhances the exploration and exploitation phases through increasing the diversity of the microbats and improving the local search. The proposed CLBAT algorithm is first tested on a set of benchmark functions to verify its accuracy and optimization stability compared to recent algorithms in the literature. CLBAT is then deployed for the optimal design of the PSS and SVC controllers for the TAFM system through optimal relocation of oscillation modes considering four operating conditions. Simulation results, eigenvalue analysis, and performance indices confirm the effectiveness of the CLBAT-based controllers in providing sufficient damping during various system disturbances across many operating cases. The future work will focus on the design of wide area controllers of a very large-scale power systems based on global signals rather than local signals.

\section{Appendix A:}

CLBAT: $\alpha=0.98, \gamma=0.98, \mathrm{~A}=1.8, r=0.8, f_{\max }=2, f_{\min }=0, W_{\max }=0.9, W_{\min }=0.2, P c_{\max }=0.8, P c_{\min }=0.2, \mathrm{G}=40$.

BA: $\alpha=0.9, \gamma=0.9, A=1, r=0.8, f_{\max }=1, f_{\min }=0$.

Excitation system: $K_{A}=155, T_{A}=0.055 \mathrm{~s}, K_{f}=0.125, T_{f}=1.8 \mathrm{~s}, K_{E}=1, T_{E}=1 \mathrm{~s}, T_{r}=0.05 \mathrm{~s}$, $S_{E}=f\left(E_{f d}\right)=0.0056\left(\mathrm{e}^{\left(1.075\left|E_{f d}\right|\right)}-1\right)$

SVC Controller: $T_{r}=0.02 \mathrm{~s}, K_{r}=10$. 


\section{References:}

Abd-Elazim, S. M., \& Ali, E. S. (2012). Coordinated design of PSSs and SVC via bacteria foraging optimization algorithm in a multimachine power system. International Journal of Electrical Power \& Energy Systems, 41(1), 44-53.

Abd-Elazim, S. M., \& Ali, E. S. (2016). Load frequency controller design via BAT algorithm for nonlinear interconnected power system. International Journal of Electrical Power \& Energy Systems, 77, 166177.

Abdelaziz, A. Y., \& Ali, E. S. (2015). Static VAR compensator damping controller design based on flower pollination algorithm for a multi-machine power system. Electric Power Components and Systems, 43(11), 1268-1277.

Abdel-Magid, Y. L., and M. A. Abido. "Optimal multiobjective design of robust power system stabilizers using genetic algorithms." IEEE Transactions on Power Systems 18.3 (2003): 1125-1132.

Abido, M. A., \& Abdel-Magid, Y. L. (2003). Coordinated design of a PSS and an SVC-based controller to enhance power system stability. International journal of electrical power \& energy systems, 25(9), 695-704.

Ali, E. S. (2014). Optimization of power system stabilizers using BAT search algorithm. International Journal of Electrical Power \& Energy Systems, 61, 683-690.

Bahmani-Firouzi, B., \& Azizipanah-Abarghooee, R. (2014). Optimal sizing of battery energy storage for micro-grid operation management using a new improved bat algorithm. International Journal of Electrical Power \& Energy Systems, 56, 42-54.

Bian, X. Y., Geng, Y., Lo, K. L., Fu, Y., \& Zhou, Q. B. (2016). Coordination of PSSs and SVC damping controller to improve probabilistic small-signal stability of power system with wind farm integration. IEEE Transactions on Power Systems, 31(3), 2371-2382. 
Bouchama, Z., Essounbouli, N., Harmas, M. N., Hamzaoui, A., \& Saoudi, K. (2016). Reaching phase free adaptive fuzzy synergetic power system stabilizer. International Journal of Electrical Power \& Energy Systems, 77, 43-49.

Castellanos, R. B., Messina, A. R., \& Sarmiento, H. U. (2008). A $\mu$-analysis approach to power system stability robustness evaluation. Electric Power Systems Research, 78(2), 192-201.

Darabian, M., Mohseni-Bonab, S. M., \& Mohammadi-Ivatloo, B. (2015). Improvement of power system stability by optimal SVC controller design using shuffled frog-leaping algorithm. IETE Journal of Research, 61(2), 160-169.

de Campos, V. A. F., da Cruz, J. J., \& Zanetta Jr, L. C. (2014). Robust control of electrical power systems using PSSs and Bilinear Matrix Inequalities. International Journal of Electrical Power \& Energy Systems, 62, 10-18.

Elazim, SM Abd, and E. S. Ali. "Optimal power system stabilizers design via cuckoo search algorithm." International Journal of Electrical Power \& Energy Systems 75 (2016): 99-107.

Eslami, M., Shareef, H., Mohamed, A., \& Khajehzadeh, M. (2012). An efficient particle swarm optimization technique with chaotic sequence for optimal tuning and placement of PSS in power systems. International Journal of Electrical Power \& Energy Systems, 43(1), 1467-1478.

Farah, Anouar, Tawfik Guesmi, and Hsan Hadj Abdallah. "A new method for the coordinated design of power system damping controllers." Engineering Applications of Artificial Intelligence 64 (2017): 325-339.

Gao, W., Chan, F. T., Huang, L., \& Liu, S. (2015). Bare bones artificial bee colony algorithm with parameter adaptation and fitness-based neighborhood. Information Sciences, 316, 180-200.

Ho, Y. C., \& Pepyne, D. L. (2002). Simple explanation of the no-free-lunch theorem and its implications. Journal of optimization theory and applications, 115(3), 549-570. 
Hussein, T., Saad, M. S., Elshafei, A. L., \& Bahgat, A. (2010). Damping inter-area modes of oscillation using an adaptive fuzzy power system stabilizer. Electric Power Systems Research, 80(12), 14281436.

Jalili, S., Hosseinzadeh, Y., \& Taghizadieh, N. (2016). A biogeography-based optimization for optimum discrete design of skeletal structures. Engineering Optimization, 48(9), 1491-1514.

Karaboga, D., \& Basturk, B. (2007). A powerful and efficient algorithm for numerical function optimization: artificial bee colony (ABC) algorithm. Journal of global optimization, 39(3), 459-471.

Khan, L., \& Lo, K. L. (2006). Hybrid micro-GA based FLCs for TCSC and UPFC in a multi-machine environment. Electric Power Systems Research, 76(9-10), 832-843.

Klein, M., Rogers, G. J., \& Kundur, P. (1991). A fundamental study of inter-area oscillations in power systems. IEEE Transactions on power systems, 6(3), 914-921.

Kundur, P., Balu, N. J., \& Lauby, M. G. (1994). Power system stability and control (Vol. 7). New York: McGraw-hill.

Liang, Jing J., et al. "Comprehensive learning particle swarm optimizer for global optimization of multimodal functions." IEEE transactions on evolutionary computation 10.3 (2006): 281-295.

Liu, Q., Wu, L., Xiao, W., Wang, F., \& Zhang, L. (2018). A novel hybrid bat algorithm for solving continuous optimization problems. Applied Soft Computing.

Mahabuba, A., \& Khan, M. A. (2009). Small signal stability enhancement of a multi-machine power system using robust and adaptive fuzzy neural network-based power system stabilizer. European Transactions on Electrical Power, 19(7), 978-1001.

Mallick, R., Ganguli, R., \& Bhat, M. S. (2015). Robust design of multiple trailing edge flaps for helicopter vibration reduction: A multi-objective bat algorithm approach. Engineering Optimization, 47(9), 1243-1263. 
Martins, N., \& Lima, L. T. G. (1989). Determination of suitable locations for power system stabilizers and static VAr compensators for damping electromechanical oscillations in large scale power systems. In Conference Papers Power Industry Computer Application Conference (Vol. 5, pp. 1455-1469)

Meng, X. B., Gao, X. Z., Liu, Y., \& Zhang, H. (2015). A novel bat algorithm with habitat selection and Doppler effect in echoes for optimization. Expert Systems with Applications, 42(17-18), 6350-6364.

Mondal, D., Chakrabarti, A., \& Sengupta, A. (2012). Optimal placement and parameter setting of SVC and TCSC using PSO to mitigate small signal stability problem. International Journal of Electrical Power \& Energy Systems, 42(1), 334-340.

Nechadi, E., Harmas, M. N., Hamzaoui, A., \& Essounbouli, N. (2012). Type-2 fuzzy based adaptive synergetic power system control. Electric Power Systems Research, 88, 9-15.

Oshaba, A. S., Ali, E. S., \& Elazim, S. A. (2017). PI controller design for MPPT of photovoltaic system supplying SRM via BAT search algorithm. Neural Computing and Applications, 28(4), 651-667.

Peres, Wesley, Ivo Chaves Silva Júnior, and João Alberto Passos Filho. "Gradient based hybrid metaheuristics for robust tuning of power system stabilizers." International Journal of Electrical Power \& Energy Systems 95 (2018): 47-72.

Saad, A., Dong, Z., Buckham, B., Crawford, C., Younis, A., \& Karimi, M. (2018). A new Kriging-Bat Algorithm for solving computationally expensive black-box global optimization problems. Engineering Optimization, 1-21

Sambariya, D. K., \& Prasad, R. (2015). Optimal tuning of fuzzy logic power system stabilizer using harmony search algorithm. International Journal of Fuzzy Systems, 17(3), 457-470.

Sauer, P. W., \& Pai, M. A. (1998). Power system dynamics and stability (Vol. 101). Upper Saddle River, NJ: Prentice hall.

Sebaa, K., \& Boudour, M. (2009). Optimal locations and tuning of robust power system stabilizer using genetic algorithms. Electric Power Systems Research, 79(2), 406-416. 
Shahgholian, G., \& Movahedi, A. (2016). Power system stabiliser and flexible alternating current transmission systems controller coordinated design using adaptive velocity update relaxation particle swarm optimisation algorithm in multi-machine power system. IET Generation, Transmission \& Distribution, 10(8), 1860-1868.

Simon, D. (2008). Biogeography-based optimization. IEEE transactions on evolutionary computation, 12(6), 702-713.

Talafuse, T. P., \& Pohl, E. A. (2016). A bat algorithm for the redundancy allocation problem. Engineering Optimization, 48(5), 900-910.

T.C.Yang,"Applying Ho optimization method to power system stabiLizer design—Parts1\&2,” Int.J.Electrical Power \& Energy Systems, vol.19,no.1,pp.29-43,1997.

Tofighi, M., Alizadeh, M., Ganjefar, S., \& Alizadeh, M. (2015). Direct adaptive power system stabilizer design using fuzzy wavelet neural network with self-recurrent consequent part. Applied Soft Computing, 28, 514-526.

Wang, D., Ma, N., Wei, M., \& Liu, Y. (2018). Parameters tuning of power system stabilizer PSS4B using hybrid particle swarm optimization algorithm. International Transactions on Electrical Energy Systems, 28(9), e2598.

Wang, G. G., \& Tan, Y. (2017). Improving metaheuristic algorithms with information feedback models. IEEE Transactions on Cybernetics.

Yang, Xin-She, and Amir Hossein Gandomi. "Bat algorithm: a novel approach for global engineering optimization." Engineering Computations 29.5 (2012): 464-483.

Zhan, Z. H., Zhang, J., Li, Y., \& Chung, H. S. H. (2009). Adaptive particle swarm optimization. IEEE Transactions on Systems, Man, and Cybernetics, Part B (Cybernetics), 39(6), 1362-13. 




Figure 1. Block diagram of PSS and excitation system.

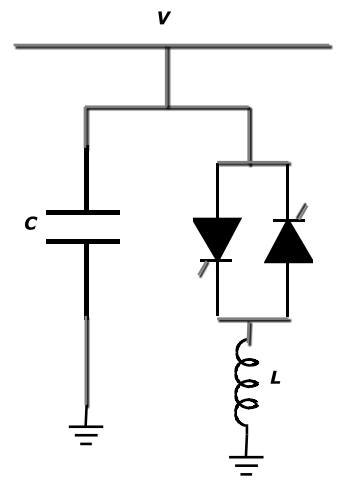

Figure 2. SVC model.



Figure 3. Block diagram of SVC.

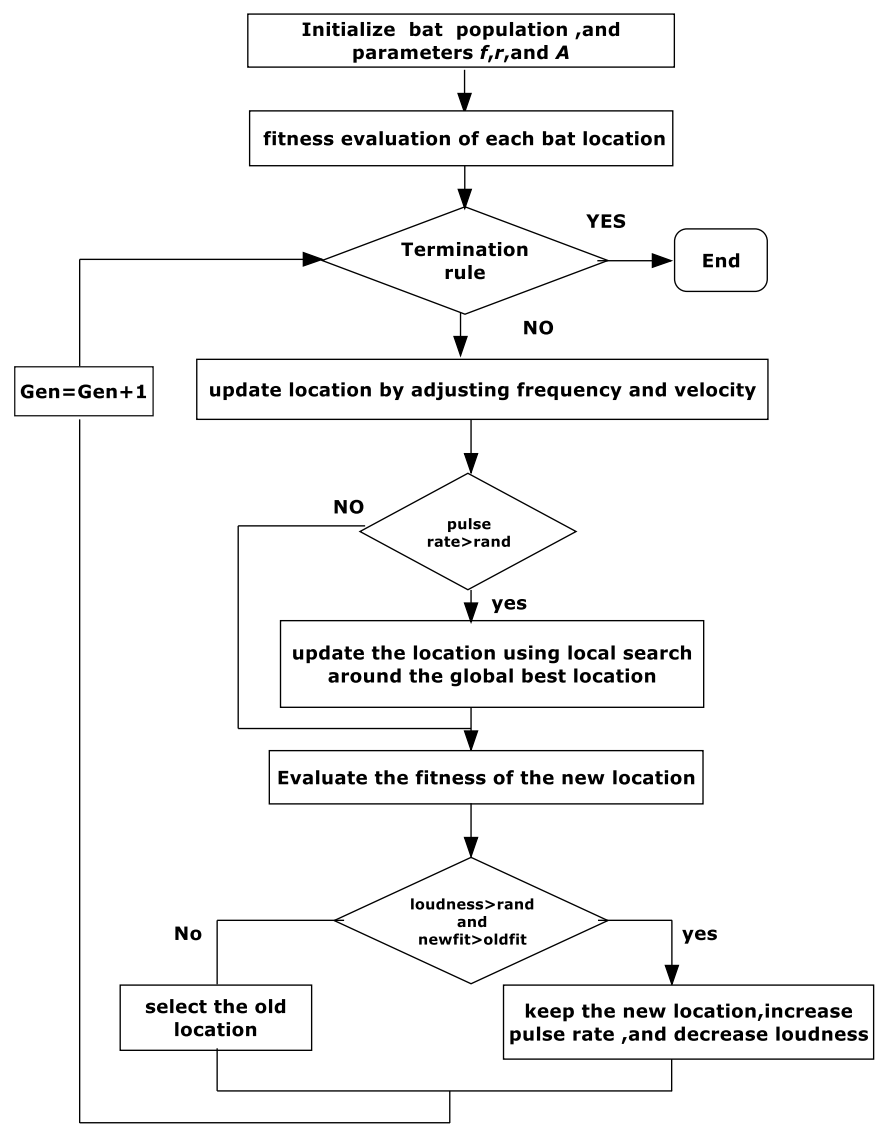

Figure 4. BA flow chart. 


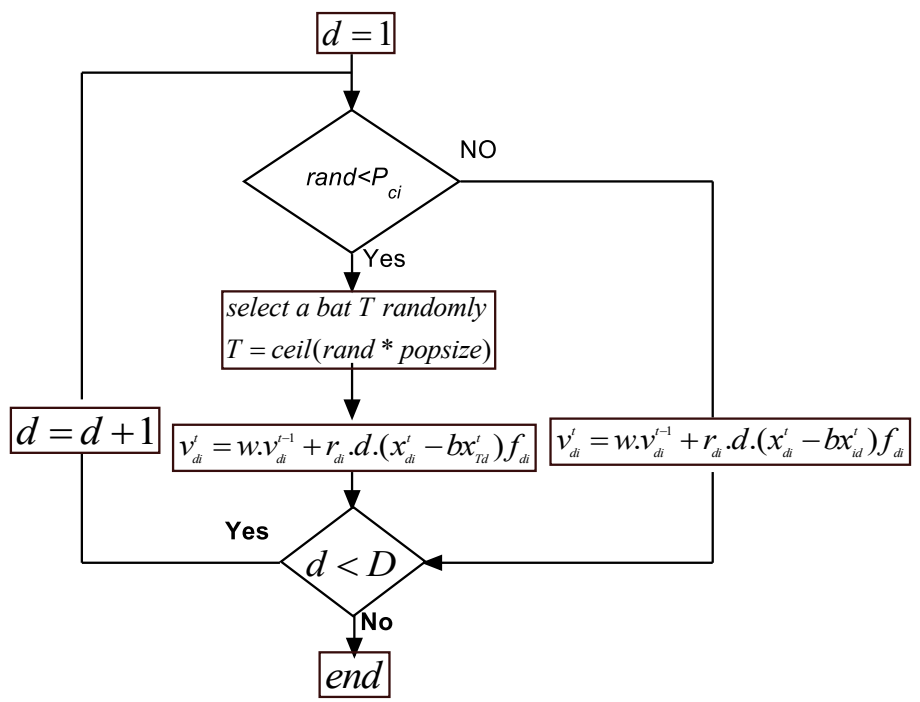

Figure 5. Velocity update for Bat $i$ at iteration $t$.

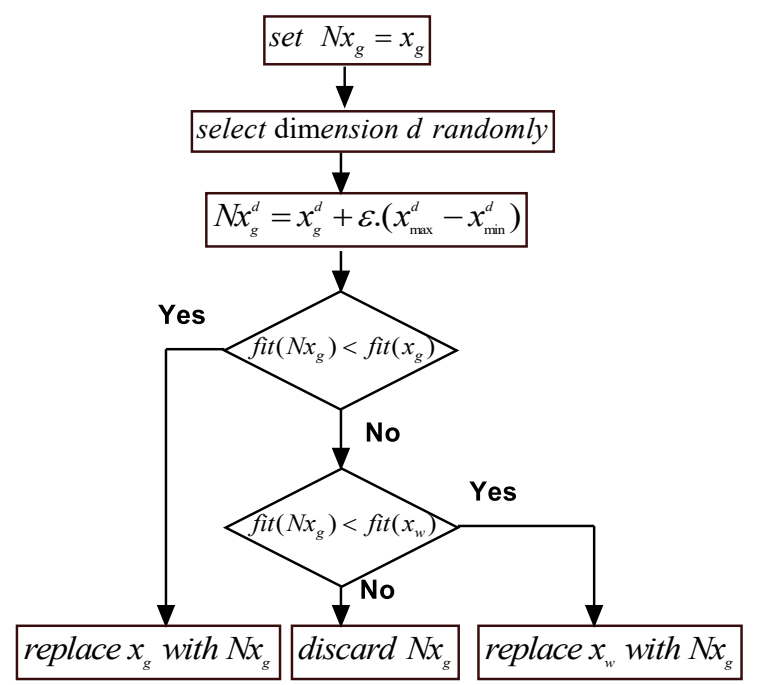

Figure 6. Modified Elitist learning strategy.


Figure 7. The D contour. Figure 8. Two Area Four machines power system.

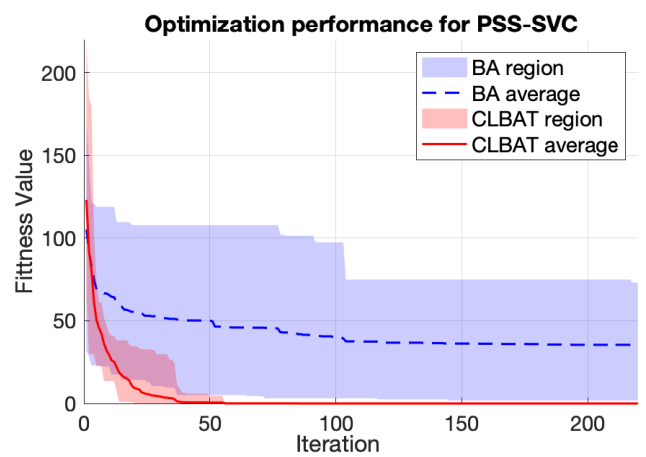

Figure 9. Convergence of CLBAT and BA algorithms. 

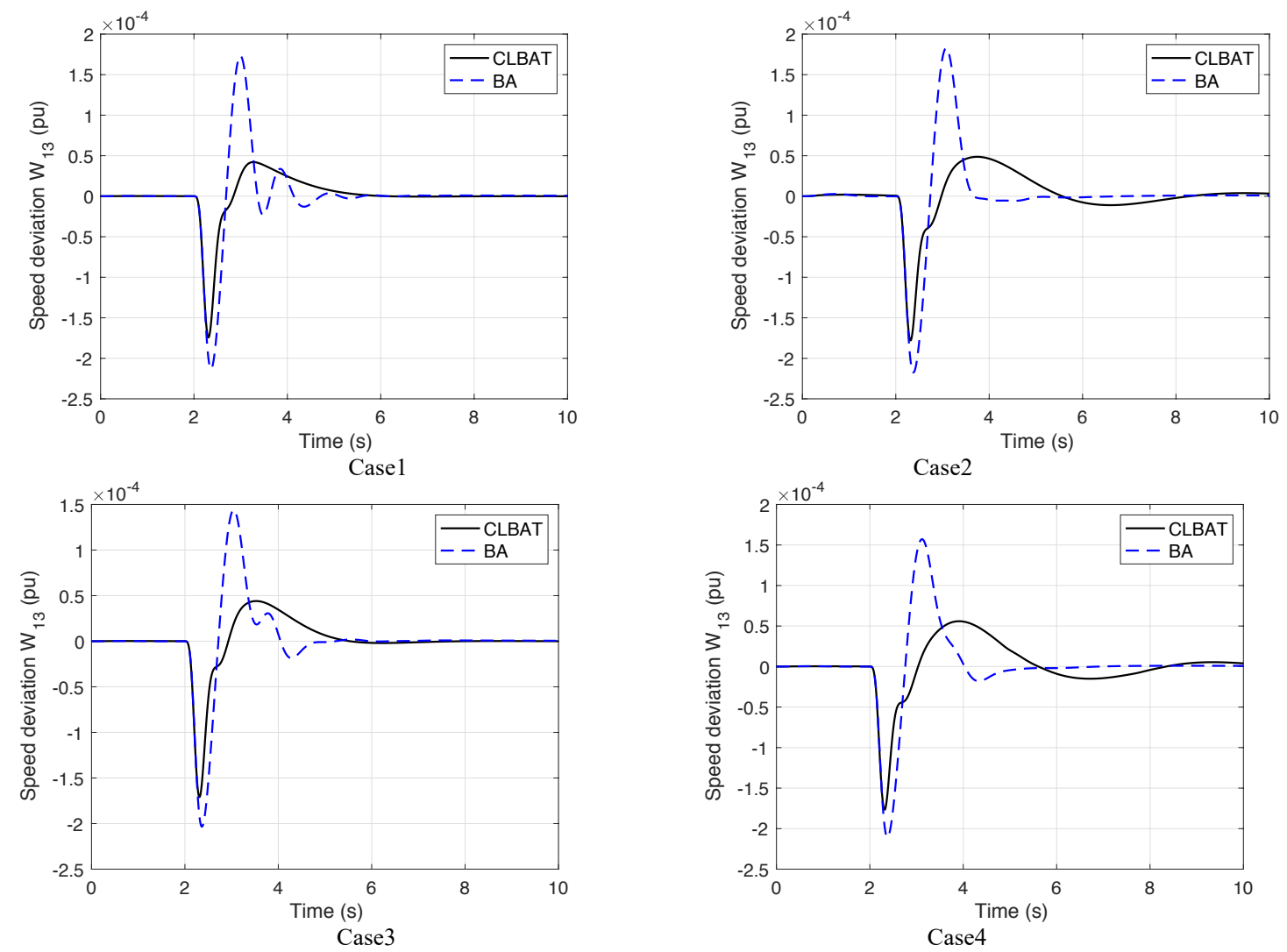

Figure 10. Response of $\omega_{13}$ for a 0.1 pu step increase in $V_{\text {ref }}$ under all cases.
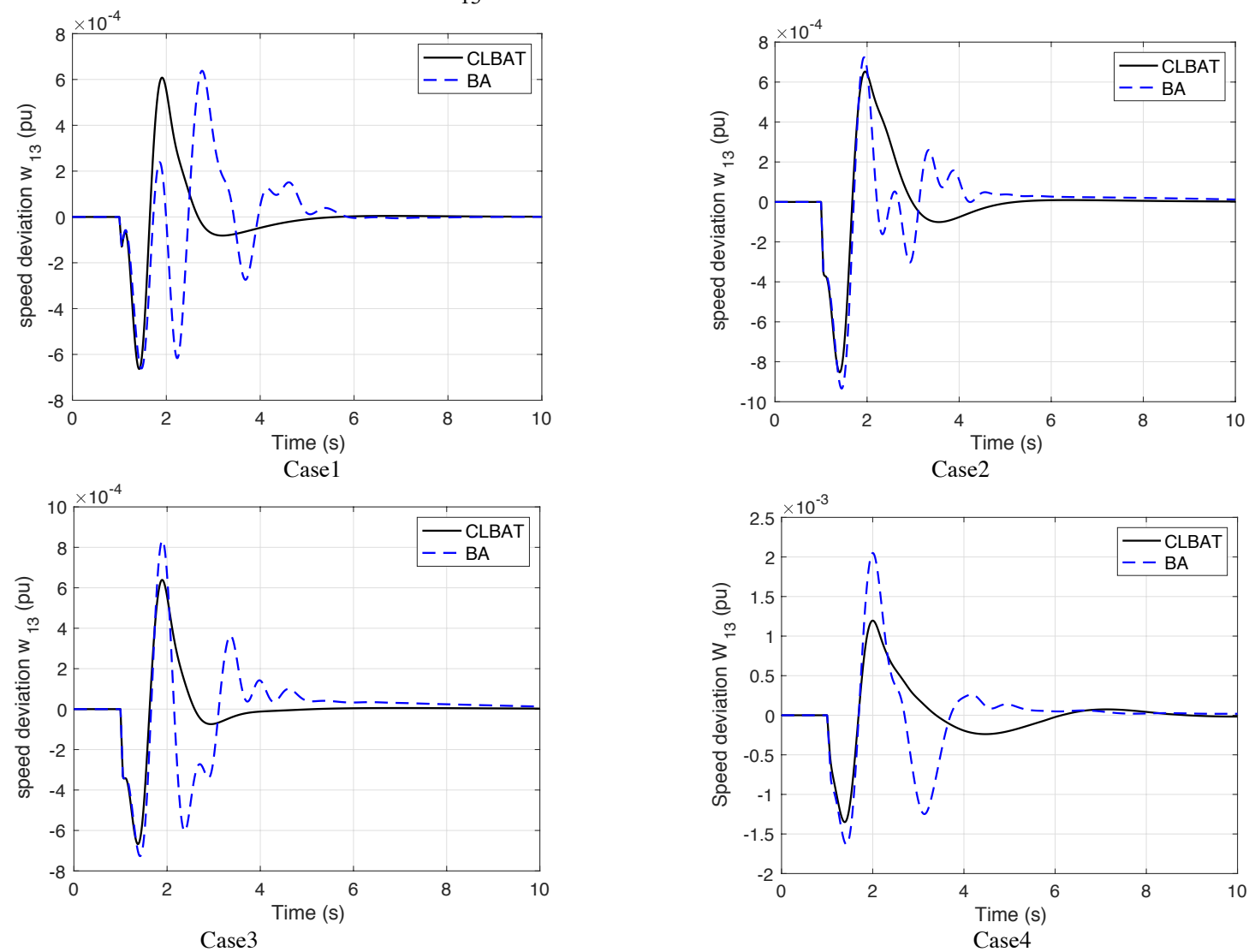

Figure 11. Response of $\omega_{13}$ for 3-cycle 3 phase fault at bus 9 under all cases. 


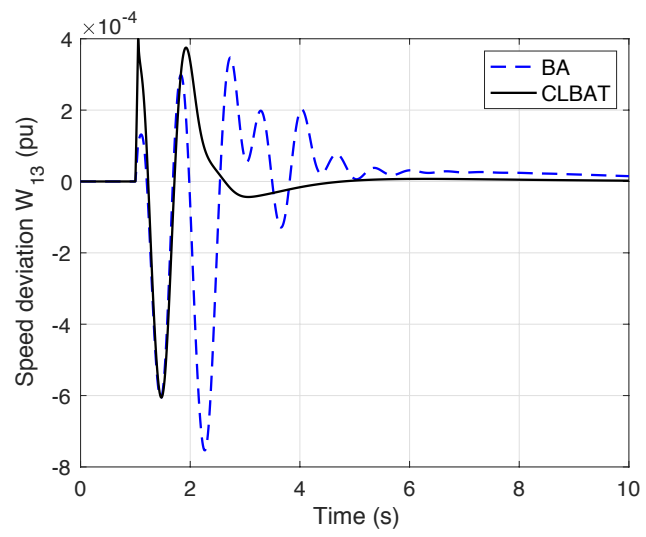

Figure 12. Response of $\omega_{13}$ for 3 -cycle 3 phase fault at bus 9 under light loading condition. 